\title{
Hemofilia A: una enfermedad huérfana
}

\section{Hemophilia A: a rare disease}

\author{
Vanessa Santiago-Pacheco ${ }^{1}$ Jennifer Vizcaíno-Carruyo²
}

Resumen. La hemofilia $A$ es una enfermedad hereditaria ligada al cromosoma $X$, causada por mutaciones en el gen F8 del factor VIII de la coagulación. Se considera una enfermedad huérfana, ya que su prevalencia es baja, de 26,6 por cada 100.000 nacidos vivos de sexo masculino. Los pacientes con hemofilia A tienen fases de inicio y amplificación de la coagulación relativamente normales y son capaces de formar el tapón plaquetario inicial en el lugar de la hemorragia, pero debido a la deficiencia del factor VIII, son incapaces de generar una cantidad de trombina en la superficie de las plaquetas, que sea suficiente para estabilizar el coágulo de fibrina. En un paciente masculino con hemorragias inusuales debe descartarse un trastorno de coagulación tipo hemofilia $A$, y se debe solicitar un recuento de plaquetas y un tiempo de protrombina (TP), los cuales usualmente son normales, y un tiempo de tromboplastina parcial activado (TPT) que se presenta prolongado. Para el diagnóstico diferencial con otras coagulopatías se realiza la medición de factores de coagulación, y pruebas de corrección cuando existe la sospecha de un inhibidor o de una hemofilia adquirida. Los pacientes afectados pueden presentar formas leves, moderadas o severas de la enfermedad, según el nivel plasmático del factor. En Colombia y en el mundo, la hemofilia fue reconocida como una enfermedad huérfana que representa un problema de salud pública, debido a su proceso de atención altamente especializado, que incrementa los costos asociados con la asistencia sanitaria, y afecta la calidad de vida de los pacientes y de aquellos que los rodean, además de que representa un reto diagnóstico que requiere constante actualización, para que pueda ser tratada de manera efectiva.

Palabras clave: enfermedad huérfana, hemofilia A, deficiencia de factor VIII, tiempo de tromboplastina parcial, aloanticuerpos, profilaxis.

\footnotetext{
${ }^{1}$ Médica, Especialista en Patología, Laboratorio Clínico Hematológico S.A. Profesora Asistente, Universidad de Antioquia. Medellín, Colombia. E-mail: vsantiago@hematologico.com.

2 Médica, Especialista en Hematología. Asistente Científica, Editora Médica Colombiana S.A. Medellín, Colombia.

Conflicto de interés: las autoras declaran que no tienen conflicto de interés.

Medicina \& Laboratorio 2021;25:605-617. https://doi.org/10.36384/01232576.452.

Recibido el 16 de mayo de 2021; aceptado el 3 de junio de 2021. Editora Médica Colombiana S.A., 2021 ${ }^{\circ}$.
} 
Abstract. Hemophilia $A$ is an X-linked inherited disease caused by mutations in the coagulation factor VIII F8 gene. It is considered a rare disease, as its prevalence is 26.6 per 100,000 live male births. Patients with hemophilia A have a relatively normal coagulation onset and amplification phases, and are able to form the initial platelet plug at the site of hemorrhage; but due to factor VIII deficiency, they are unable to generate a sufficient amount of thrombin on the platelet surface to stabilize the fibrin clot. In a male patient with unusual bleeding, a hemophilia A-type coagulation disorder should be ruled out, and blood tests such as a platelet count and prothrombin time (PT), which are usually normal, and an activated partial thromboplastin time (APTT), which is prolonged, should be requested immediately. For differential diagnosis with other coagulopathies, measurement of coagulation factors and correction tests are performed when there is suspicion of an inhibitor or acquired hemophilia. Affected patients may present mild, moderate or severe forms of the disease, depending on the plasma level of the factor. In Colombia and worldwide, hemophilia was recognized as a rare disease that represents a public health problem due to its highly specialized care, which increases the costs associated with health care, and affects the quality of life of patients and those around them, as well as representing a diagnostic challenge that requires constant updating, so that it can be treated effectively.

Keywords: rare diseases, hemophilia A, factor VIII deficiency, partial thromboplastin time, alloantibodies, prophylaxis.

\section{Introducción}

La hemofilia es una enfermedad hereditaria ligada al cromosoma $\mathrm{X}$, causada por mutaciones en los genes de los factores VIII (hemofilia A) y IX (hemofilia B) de la coagulación, asociada a sangrado [1]. Los pacientes afectados pueden presentar formas leves, moderadas 0 severas de la enfermedad, según el nivel plasmático del factor. A pesar de ser muy reconocida por el público general, se considera una enfermedad huérfana en todo el mundo [2], ya que su prevalencia es baja, de 26,6 por cada 100.000 nacidos vivos de sexo masculino $[3,4]$. El número de pacientes con hemofilia en todo el mundo es de 1.125.000, de los cuales 418.000 tendrían hemofilia severa [3]; también se sabe que la hemofilia A es mucho más frecuente que la hemofilia $B, 80 \%$ a $85 \%$ versus $15 \%$ a $20 \%$, respectivamente [5].
En un principio se pensaba que la tendencia hemorrágica de la hemofilia se debía a fragilidad de los vasos sanguíneos; en los años 30, se propuso que estaba asociada a alteraciones en las plaquetas. En 1937, se encontró que al adicionar una sustancia, que en ese momento fue llamada "globulina antihemofílica", a la sangre del paciente, se corregía el defecto de la coagulación. Posteriormente, en 1944, las observaciones de Pavlosky mostraron que la sangre de un paciente con hemofilia corregía el defecto de la coagulación de otro paciente, y viceversa, demostrando el defecto en dos factores distintos de la coagulación: FVIII y FIX [6]. Estos hallazgos permitieron un diagnóstico preciso y el desarrollo de un tratamiento apropiado para esta enfermedad.

La hemofilia A se hereda de forma recesiva, y el riesgo para los hermanos 
de un probando depende del estado de portador de la madre. Las mujeres portadoras tienen un $50 \%$ de posibilidad de transmitir la variante patógena del gen F8 en cada embarazo, por lo tanto, los hijos que la hereden estarán afectados y las hijas que la hereden serán portadoras; por otro lado, los varones afectados transmiten la variante patógena a todas sus hijas y a ninguno de sus hijos [7]. Las mujeres y niñas declaradas como "mujeres hemofílicas" pueden tener causas genéticas complejas para expresar el fenotipo de hemofilia, tales como homocigosidad (dos alelos de hemofilia idénticos), heterocigosidad compuesta (dos alelos de hemofilia diferentes), hemicigosidad (un alelo de hemofilia y ningún alelo normal), heterocigosidad (un alelo de hemofilia y un alelo normal), causas genéticas distintas de la hemofilia y causas no genéticas. Los estudios necesarios para la clasificación pueden incluir estudios de inactivación del cromosoma $X$ realizados en el paciente y los padres. Las mujeres y las niñas pueden tener hemorragias excesivas que requieren tratamiento, simplemente porque son heterocigotas para los alelos de la hemofilia [8].

En esta revisión se analizarán los aspectos más resaltantes de la hemofilia A, como su etiología y fisiopatología, características clínicas, diagnóstico, tratamiento y complicaciones, y por último se abordará su problemática en Colombia.

\section{Etiología y fisiopatología}

Para mantener la hemostasia, el cuerpo humano tiene un sistema complejo que permite el balance de los estados procoagulante, anticoagulante y fibrinolítico, para mantener la fluidez de la sangre dentro del sistema vascular y lograr desarrollar un trombo rápidamente como respuesta a lesiones vasculares. En este proceso, es esencial la activación secuencial de los complejos compuestos por factores dependientes de vitamina $\mathrm{K}$ (FVII, FIX, FX) y sus respectivos cofactores (factor tisular, FVIII, FV) [9].

El modelo clásico de coagulación que separa las vías intrínseca (activada por factores de contacto) y extrínseca (mediada por el factor tisular) es útil para fines didácticos y para valorar resultados de los estudios de laboratorio [1]; sin embargo, in vivo, ambos procesos son simultáneos, por lo que actualmente se le da el nombre de modelo celular de la coagulación, donde el factor VII activado (FVIla) por el factor tisular, no solo activa el factor $X(F X)$, sino también el factor IX (FIX). Posteriormente, el FXa puede activar tanto el FVIII como el FV. Asimismo, la trombina juega un papel fundamental, amplificando todo el proceso. Según este modelo, la coagulación está esquematizada en las siguientes fases: 1) iniciación, la cual se da en la superficie de la célula portadora del factor tisular (FT); 2) amplificación, con la activación de las plaquetas y de los cofactores para poder generar mayores cantidades de trombina; $y, 3$ ) propagación, donde se da la generación de grandes cantidades de trombina (Ila) en la superficie plaquetaria [10] (figura 1).

Los pacientes con hemofilia A tienen fases de inicio y amplificación de la coagulación relativamente normales, y son capaces de formar el tapón plaquetario inicial en el lugar de la hemorragia; sin embargo, son incapaces de generar una cantidad de trombina en la superficie de las plaquetas que sea suficiente (fase 3 de propagación) para estabilizar el coágulo de fibrina, debido a la deficiencia del factor VIII [11]. 


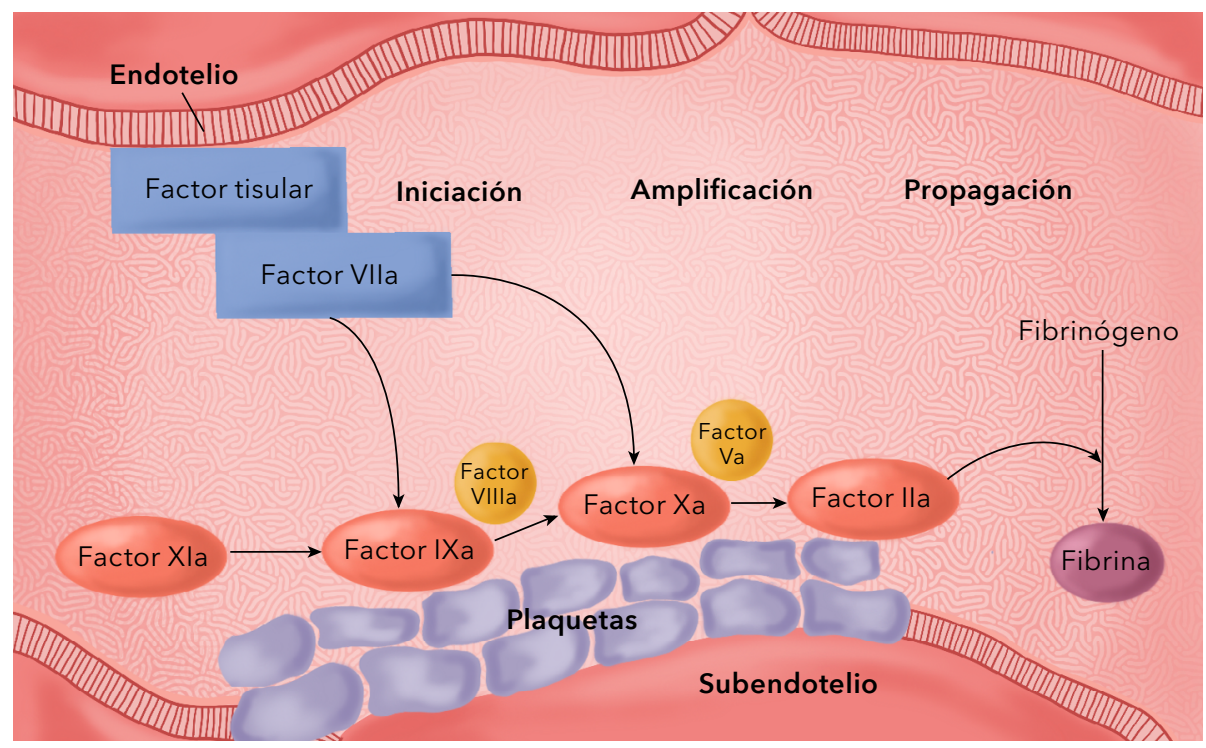

Figura 1. Modelo celular de la coagulación.

\section{Características clínicas}

Según la actividad residual del factor afectado, la hemofilia A se clasifica en leve, moderada o severa [12,13]; de esto depende la edad de inicio de los síntomas, el tipo de síntomas, la tasa de sangrados intraarticulares espontáneos y el desarrollo de inhibidores (tabla 1).

En la presentación clínica de la hemofilia A, las articulaciones son el principal sitio de sangrado espontáneo, principalmente el tobillo, seguido de los codos y las rodillas. Aproximadamente la mitad de los niños con hemofilia severa desarrollan hematomas intramusculares desde los 6 a 8 meses de edad [14]. Los pacientes con hemofilia leve suelen sangrar excesivamente únicamente en asociación a cirugía o trauma mayor, sin embargo, aquellos con hemofilia severa presentan episodios frecuentes de sangrado espontáneo, especialmente intraarticular o muscular, luego de traumas menores [15].
El sangrado articular recurrente induce una cascada de procesos inflamatorios y degenerativos que lesionan la sinovia, el cartílago y el hueso. El mayor detonante de estos procesos es el hierro liberado al líquido sinovial, que tiene actividad proinflamatoria y angiogénica; la neovascularización asociada lleva a la formación de nuevos vasos friables, más propensos al sangrado, lo que lleva a un ciclo de sangrado, acumulación de hierro, hipertrofia sinovial y nuevo sangrado [16]. Debido a estos ciclos, muchos pacientes desarrollan sinovitis crónica con edema articular; otros llegan a la artropatía hemofílica con daño osteocondral severo. Esta artropatía lleva a dolor crónico, pérdida del rango de movimientos, atrofia muscular y, debido a esto, reducción de la calidad de vida [17]. Otra complicación frecuente es la hemorragia intracraneana, con una incidencia del $1,9 \%$ y una tasa de mortalidad del $19,6 \%$ [18]. 


\begin{tabular}{|c|c|c|c|}
\hline & Leve & Moderada & Severa \\
\hline $\begin{array}{l}\text { Actividad residual } \\
\text { del factor }\end{array}$ & $5 \%$ a $40 \%$ & $1 \%$ a $5 \%$ & $<1 \%$ \\
\hline Presentación clínica & $\begin{array}{l}\text { - Sangrado } \\
\text { postraumático o } \\
\text { postquirúrgico }\end{array}$ & $\begin{array}{l}\text { - Historia familiar } \\
\text { - Sangrado neonatal } \\
\text { (menos frecuente) } \\
\text { - Sangrado asociado } \\
\text { a vacunación } \\
\text { - Sangrado por } \\
\text { mucosas } \\
\text { - Sangrado articular }\end{array}$ & $\begin{array}{l}\text { - Historia familiar } \\
\text { - Sangrado neonatal } \\
\text { (circuncisión) } \\
\text { - Sangrado asociado a } \\
\text { vacunación } \\
\text { - Hematomas en } \\
\text { menores de } 1 \text { año } \\
\text { - Sangrado por } \\
\text { mucosas } \\
\text { - Sangrado articular }\end{array}$ \\
\hline Edad de inicio & $\begin{array}{c}>5 \text { años y adultos } \\
\text { jóvenes }\end{array}$ & $\begin{array}{l}\text { Desde el nacimiento a } \\
\text { los } 10 \text { años }\end{array}$ & $\begin{array}{l}\text { Desde el nacimiento a } \\
\text { los } 3 \text { años }\end{array}$ \\
\hline $\begin{array}{l}\text { Riesgo de desarrollo } \\
\text { de inhibidores }\end{array}$ & Escaso & $1 \%$ a $2 \%$ & $\begin{array}{l}25 \% \text { en hemofilia A } \\
5 \% \text { en hemofilia B }\end{array}$ \\
\hline $\begin{array}{l}\text { Riesgo de artropatía } \\
\text { hemofílica }\end{array}$ & Escaso & Común sin profilaxis & $\begin{array}{c}\text { Ocurrencia universal sin } \\
\text { profilaxis }\end{array}$ \\
\hline
\end{tabular}

\section{Diagnóstico}

El diagnóstico de la hemofilia $A$ es relativamente sencillo y debe sospecharse siempre que se produzcan hemorragias inusuales en un paciente masculino. Las pruebas de laboratorio usualmente muestran un recuento de plaquetas y un tiempo de protrombina (TP) normales, pero un tiempo de tromboplastina parcial activado (TPT) prolongado; no obstante, se recomienda que un resultado de TPT dentro del rango de referencia no se utilice para descartar la presencia de hemofilia, ya que en algunos casos de hemofilia $A$ leve, este puede estar dentro del rango normal [5].

Dado que las hemofilias A y B son clínicamente similares, se deben realizar pruebas específicas de medición de la actividad de los factores VIII y IX. Para los pacientes con sospecha clínica de hemofilia A, la Federación Mundial de Hemofilia (FMH) recomienda el uso tanto de la prueba de FVIII de una etapa, como la prueba cromogénica de FVIII:C en la evaluación diagnóstica inicial [5]. La confirmación se da cuando el nivel de actividad del factor es inferior al normal en dos determinaciones analíticas ( $<50 \%$ o $50 \mathrm{UI} / \mathrm{dL}$ ), tanto por déficit de la proteína, como por alteración de su función [10]. Los pacientes con la enfermedad de von Willebrand, también tienen una deficiencia del factor VIII secundaria a una deficiencia del factor de von Willebrand, por lo que es importante diferenciarlas [19].

Cuando una prueba de TPT es anormal, se puede investigar mejor utilizando estudios de mezcla o corrección con un pool de plasma normal, que puede ayudar a definir si las alteraciones se deben a la deficiencia de factor, a uso de anticoagulantes o presencia de inhibidores circulantes. La prueba de TPT se prolonga sustancialmente en un período de 1 a 2 horas de incubación a $37^{\circ} \mathrm{C}$, si la muestra del pacien- 
te contiene un inhibidor neutralizante anti-FVIII [5]. Se debe tener en cuenta que al contrario de la hemofilia $B$, la hemofilia A sí se puede diagnosticar al nacer, incluso con una muestra de sangre del cordón umbilical [20], ya que el factor VIII no debe encontrarse en niveles bajos al nacer, lo que sí ocurre con el factor IX.

Es importante señalar que el modelo clásico de la cascada de coagulación y las pruebas de coagulación comunes no reflejan la complejidad de la hemostasia in vivo, por lo que no pueden caracterizar la verdadera gravedad del fenotipo de la hemofilia A, no obstante, sí tienen la sensibilidad para detectar deficiencias de uno o más factores de coagulación. A pesar de que ninguna prueba es capaz de proporcionar un perfil completo y fiable de la función hemostática [11], han surgido pruebas que evalúan globalmente el proceso de coagulación, como la tromboelastografía (TEG) o la tromboelastometría rotacional (ROTEM), que dependen de la actividad del sistema de coagulación del plasma, de la función de las plaquetas y de la fibrinólisis; la prueba de generación de trombina (TGA), que refleja la interacción de procoagulantes y anticoagulantes selectos; y, el análisis de la forma de onda del coágulo, que se deriva de la medición constante de la transmitancia de la luz, tomada durante los ensayos rutinarios de TPT [13]. Estas pruebas han demostrado proporcionar información multilateral sobre las propiedades de la coagulación, en comparación a una simple prueba de coagulación intrínseca como lo es el TPT [21].

En general y para aquellos pacientes que se encuentran en países en vías de desarrollo, y que no tienen acceso a métodos de diagnóstico de calidad para hemostasia, se ha sugerido un nuevo esquema de diagnóstico que incluye una herramienta para la evaluación de hemorragias (BAT, del inglés, Bleeding Assessment Tool) [22], desarrollada por la Sociedad Internacional de Trombosis y Hemostasia (ISTH), seguida de una toma de muestra solo para los que tengan un puntaje más elevado, que se envía a un laboratorio de genómica bien establecido en cualquier parte del mundo, para un diagnóstico genético basado en secuenciación de nueva generación (NGS) [23]; esto ha demostrado ser útil para identificar a los pacientes adultos y niños con hemofilia $[23,24]$.

\section{Estudio genético}

La identificación de la mutación genética presente es esencial, ya que puede pronosticar el riesgo de formación de inhibidores [25], así como identificar mujeres portadoras de la enfermedad en la familia. Del $40 \%$ al $45 \%$ de los pacientes con hemofilia $A$ severa tienen inversión del intrón 22 del gen $F 8$, mientras que del $1 \%$ al $6 \%$, inversión del intrón 1. Al ser estas las mutaciones más comunes, se hace una búsqueda inicial de las mismas, con posterior secuenciación completa del gen, si no se encuentran. En casos de hemofilia A leve y moderada, se debe hacer secuenciación completa del gen F8 [26].

En general, todos los varones con una variante patogénica $F 8$ estarán afectados, y tendrán aproximadamente la misma gravedad de la enfermedad que otros varones afectados de la familia; sin embargo, otros factores genéticos y ambientales pueden modificar la gravedad clínica en cierta medida $[7,27]$. Las variantes nulas, aquellas que dan lugar a la ausencia total de la proteína (grandes deleciones, duplicaciones, inserciones, inversiones, mutaciones sin sentido y variantes del sitio de empal- 
me), han mostrado tener mayor asociación con el desarrollo de inhibidores en hemofilia $A$, en comparación con otras variantes (deleciones pequeñas dentro del marco, duplicaciones, inserciones, mutaciones con cambio de sentido) [5]. Aproximadamente el 30\% de las mujeres con una variante patogénica del $F 8$ y un alelo normal, tienen una actividad de coagulación del factor VIII inferior al $40 \%$ y un trastorno hemorrágico; pueden producirse hemorragias leves en las mujeres heterocigotas con una actividad del factor VIII baja y normal [28], pero en general, las portadoras tienen más hemorragias que las mujeres no afectadas [29].

\section{Evolución histórica del tratamiento}

En los años 50 y 60, los pacientes con hemofilia eran tratados con sangre o plasma fresco únicamente; sin embargo, debido a que la baja concentración de los factores de coagulación requeridos en estos productos no era suficiente para tratar sangrados severos, un gran número de pacientes moría en la infancia o juventud [30].

En 1964, se descubrió que la fracción crioprecipitada del plasma contiene grandes cantidades de FVIII. Esto permitió concentrar suficiente cantidad del factor en volúmenes menores y la realización de cirugías mayores; sin embargo, la era moderna del tratamiento de la hemofilia inició en los años 70 con la producción de concentrados liofilizados de factores de coagulación. Este avance permitió mejorar la calidad y prolongar la expectativa de vida de los pacientes, debido a la implementación y difusión de la terapia de reemplazo en casa, el control temprano de hemorragias y la disminución del daño músculo-esquelético asociado al tratamiento inadecuado [31]. La profilaxis primaria logró prevenir la mayoría de los episodios de sangrado articular espontáneo, disminuyendo la artropatía. Por otro lado, el descubrimiento de la desmopresina, que acorta el TPT prolongado y el tiempo de sangrado, favoreciendo la elevación del FVIII y del factor de von Willebrand, ha logrado ser un tratamiento económico y seguro para los pacientes con hemofilia A leve [32].

Desafortunadamente, la era de los concentrados de plasma para el tratamiento de la hemofilia sufrió un gran revés, debido a la transmisión del virus de la inmunodeficiencia humana $(\mathrm{VIH})$ y de la hepatitis $\mathrm{C}(\mathrm{VHC})$, a través de los concentrados de factores de coagulación fabricados a partir de plasma de múltiples donantes [30,33]. Miles de pacientes con hemofilia fallecieron por complicaciones del VIH/SIDA en los 80 y 90. Como consecuencia de esto, se buscaron tratamientos más seguros y se implementaron técnicas de inactivación viral en la producción de los factores concentrados derivados del plasma, y de tamización viral en sangre donada; todo esto aumentó la seguridad de dichos derivados [30].

El mayor avance en el tratamiento de las hemofilias se dio en las últimas décadas del siglo $\mathrm{XX}$ y tuvo que ver con el progreso de la tecnología del ADN recombinante, lo que permitió el desarrollo de FVIII y FIX recombinantes y disminuyó, así, el riesgo de transmisión de patógenos [34]. Como resultado del progreso en la terapia para la hemofilia, la esperanza de vida de los pacientes se ha equiparado a la de la población general $[35,36]$. Esto ha llevado al desarrollo de enfermedades crónicas asociadas a la edad, previamente no vistas en la población con hemofilia, como trastornos cardiovasculares y osteoporosis [33,35]. 
Entre las aproximaciones terapéuticas recientes, también se encuentran anticuerpos específicos que simulan la función coagulante del FVIII [37], inhibición de proteínas anticoagulantes, como la antitrombina, con moléculas de interferencia del ARN, y la vía inhibidora del factor tisular con anticuerpos monoclonales [38-40].

Por otro lado, se encuentran en curso estudios en fase III de terapia génica, como opción curativa contra las hemofilias [41]. La meta de la terapia génica es que los pacientes no requieran terapia de reemplazo de factores de coagulación y no presenten sangrados [42]. La terapia génica exitosa resulta en la expresión endógena del factor de coagulación, llegando a un nivel estable y con una duración de acción sostenida. Esto haría innecesarias la profilaxis y las infusiones intravenosas, además, se ha postulado que la expresión endógena de los factores podría ser menos inmunogénica, disminuyendo o evitando la generación de inhibidores [43].

\section{Inhibidores}

En el seguimiento de los pacientes es esencial valorar la presencia de inhibidores. El desarrollo de inhibidores es, en la actualidad, la complicación más difícil derivada del tratamiento con factores de coagulación. Estos son aloanticuerpos que se presentan hasta en $20 \%$ al $30 \%$ de los pacientes con hemofilia A [44], lo que hace poco eficiente la infusión de FVIII concentrado, aumenta la morbimortalidad de los pacientes y disminuye su calidad de vida. La formación de estos inhibidores es un proceso complejo y multifactorial; se ha sugerido en algunos estudios que puede variar según el tipo de producto utilizado en el tratamiento (FVIII derivado del plasma versus FVIII re- combinante), sin embargo, la evidencia al respecto no es concluyente.

Los inhibidores son anticuerpos IgG policlonales de alta afinidad dirigidos contra la proteína del FVIII y pueden ser de dos tipos: los tipo 1, que inactivan completamente el FVIII, y los tipo 2, que lo hacen de manera incompleta. Los pacientes con altos títulos de anticuerpos desarrollan complicaciones hemorrágicas severas que no responden al reemplazo de factores de coagulación [45]. Aunque muchos de estos inhibidores son transitorios, o se resuelven con inmunoterapia, hasta el $15 \%$ de los pacientes con hemofilia A presentan anticuerpos persistentes [46].

Las opciones para la erradicación del inhibidor incluyen la inducción de la tolerancia inmunológica (ITI), los agentes inmunosupresores, o la observación evitando la reexposición a los concentrados de factor VIII. En 2018, el emicizumab fue aprobado con base en los resultados del ensayo clínico HAVEN 1 para personas con hemofilia $A$ e inhibidores, con la finalidad de prevenir o reducir la frecuencia de los episodios de sangrado. Debido a que el ensayo se llevó a cabo en personas con hemofilia A severa, aún no se ha demostrado si el emicizumab es igual de efectivo en la erradicación de los inhibidores en la población con hemofilia A leve y moderada $[47,48]$.

\section{Diagnóstico diferencial}

El diagnóstico diferencial de la hemofilia A incluye todas las patologías que cursan con sangrado inexplicable, como desórdenes de plaquetas, enfermedad de von Willebrand y deficiencia de fibrinógeno; asimismo, la deficiencia de diferentes factores de la coagulación, como el factor $\mathrm{V}$, el factor VII, el 
factor $X$ y el XIII, que también cursan con hemorragias [49].

El principal diagnóstico diferencial de la hemofilia $A$ es la $B$, se cree que el tipo de sangrado es similar en ambas hemofilias, pero la mayor prevalencia de mutaciones que son menos graves (mutaciones con cambio de sentido) en hemofilia $B$, podría proporcionar una base biológica para un fenotipo hemorrágico más leve en comparación con la hemofilia $A$, aunque la evidencia es limitada [27]. La deficiencia de factor XI o también Ilamada hemofilia $C$ para diferenciarlo de las otras hemofilias, cursa con sangrados provocados y no espontáneos a diferencia de las anteriores [50], además, se presenta en proporciones similares en hombres y mujeres.

Entre otros diagnósticos diferenciales, es importante mencionar la hemofilia A adquirida, un raro trastorno hemorrágico causado por autoanticuerpos neutralizantes contra el factor VIII de la coagulación, que se presenta tanto en hombres como en mujeres sin antecedentes de hemorragia. Los pacientes suelen presentar un TPT prolongado, y los anticuerpos neutralizantes (inhibidores) se detectan mediante la prueba de Bethesda modificada por Nijmegen [51]. En pacientes con hemorragias se recomienda el tratamiento con agentes de derivación, incluyendo el factor VII activado recombinante, el concentrado de complejo de protrombina activado o el FVIII porcino recombinante; asimismo, la erradicación de los autoanticuerpos puede lograrse con un tratamiento inmunosupresor, que incluye corticosteroides, ciclofosfamida y rituximab, o combinaciones de los mismos [51]; recientemente se ha sugerido el uso de emicizumab, pero esto necesita ser validado por ensayos que incluyan un número adecuado de pacientes [52].

\section{Hemofilia en Colombia}

En el país, la hemofilia, al estar en el censo de enfermedades huérfanas, está listada como de reporte obligatorio en el Sistema Nacional de Vigilancia en Salud Pública-SIVIGILA; asimismo, la Liga Colombiana de Hemofílicos interviene, mediante el activismo y representación ante las sociedades científicas, para que las decisiones y las guías nacionales de manejo tengan en cuenta el beneficio integral de los pacientes [53].

En Colombia, para el 2017 estaban registradas 2.170 personas con hemofilia, 1.794 presentaban hemofilia $A$ y 376 hemofilia $B$, de las cuales 1.167 estaban clasificadas como hemofilia severa. De estos pacientes, 1.284 se encontraban en esquema de profilaxis y 324 (14,9\% del total) tuvieron presencia de inhibidores. En ese mismo período, los pacientes con hemofilia fallecidos fueron 16; sin embargo, únicamente dos de ellos por complicaciones asociadas a la enfermedad [54]. En otro estudio reportado por Sánchez-Duque y colaboradores para el mismo año [55], también se identificó una mayor población de pacientes con hemofilia A $(97,9 \%)$, $y$ de los que presentaron hemofilia severa $(41,7 \%)$, el $30 \%$ desarrolló inhibidores. Para el 2019, la Federación Mundial de la Hemofilia reportó que en Colombia había 2.918 pacientes con hemofilia A, 291 (10\%) con presencia de inhibidores, predominando la población de 19 a 44 años (60\%), y 45,5\% con hemofilia severa [3].

En los últimos años se ha observado un incremento en el número de pacientes con hemofilia, por lo que recientemente se publicó el Registro Nacional de Hemofilia y otras Coagulopatías, como una iniciativa multisectorial para centralizar la información sociodemográfica, clínica y económica de estos 
pacientes, por medio de un consenso de expertos, con el objetivo de monitorizar la morbilidad y mortalidad, evaluar el acceso a los servicios de salud, su impacto en las complicaciones de la enfermedad y los costos asociados a la asistencia médica. Se espera con este registro guiar la toma de decisiones racionales para un uso eficiente de los recursos económicos, además de impulsar la investigación en salud para mejorar la calidad de vida y disminuir las discapacidades asociadas en los pacientes con hemofilia [2].

En la actualidad, la calidad de vida de los niños con hemofilia ha mejorado gracias a la profilaxis y la posibilidad de realizar el tratamiento en casa; sin embargo, esta no está globalmente implementada y no ha sido ejecutada satisfactoriamente en Latinoamérica [56], por lo que el Grupo Latinoamericano para el Impulso del Tratamiento de la Hemofilia (GLAITH), recomienda el establecimiento de un registro latinoamericano unificado, así como estudios prospectivos de costo-efectividad y evaluación de criterios especialmente relacionados con profilaxis secundaria, al igual que realizar estudios comparativos de calidad de vida con y sin profilaxis en la región, promover la individualización del tratamiento, y buscar la implementación de profilaxis primaria y secundaria en forma global en Latinoamérica [56].

\section{Conclusión}

Las enfermedades huérfanas se definen como afecciones crónicas y potencialmente mortales con una baja prevalencia. En Colombia y en el mundo, la hemofilia fue reconocida como un problema de salud pública crucial debido a su proceso de atención altamente especializado, que incrementa la economía y costos asociados con la asistencia sanitaria, asimismo, esta es considerada una entidad potencialmente mortal, que afecta la calidad de vida de los pacientes y de aquellos que los rodean, además de que representa un reto diagnóstico que requiere constante actualización, para que pueda ser tratada de manera efectiva.

A pesar de los tratamientos profilácticos disponibles, los pacientes continúan teniendo muchas dificultades, entre ellas la práctica de algunos deportes, lo que genera un impacto sobre su desarrollo psicosocial. Este aspecto puede tener alguna mejoría si se logra capacitar a los pacientes para que tengan autonomía en el manejo de algunas de estas situaciones [57]. A futuro, se espera contar con incremento en la producción de concentrados de FVIII y FIX para suplir a países en vías de desarrollo, además del procesamiento de moléculas con mayor vida media y menor inmunogenicidad [31]. Adicionalmente, se recomienda fomentar las investigaciones relacionadas con el desarrollo de nuevos productos terapéuticos o incluso terapias más avanzadas, que se conviertan no solo en una herramienta de tratamiento, sino también en una herramienta curativa, como promete ser la terapia génica.

\section{Referencias}

1. Acosta-Aragón MA, Álvarez-Mina $A R$, Velásquez-Paz JC, Vizcaíno-Carruyo JC. Hemofilia B o enfermedad de Christmas. Med Lab 2020;24:273-289. https://doi. org/10.36384/01232576.336.

2. Alvis LF, Sánchez P, Acuña L, Escobar G, Linares A, Solano $\mathbf{M H}$, et al. National registry of haemophilia and other coagulopathies: A multisector initiative in the Colombian Health System. Haemophilia 2020;26:e254-261. https:// doi.org/https://doi.org/10.1111/hae.14138. 
3. World Federation of Hemophilia (WFH). Report on the Annual Global Survey 2019. Montréal, Canada: World Federation of Hemophilia; 2020. Acceso 11 de mayo de 2021. Disponible en http:// www1.wfh.org/publications/files/pdf-1806.pdf.

4. Iorio A, Stonebraker JS, Chambost H, Makris M, Coffin D, Herr C, et al. Establishing the prevalence and prevalence at birth of hemophilia in males: A meta-analytic approach using national registries. Ann Intern Med 2019;171:540546. https://doi.org/10.7326/m19-1208.

5. Srivastava A, Santagostino E, Dougall A, Kitchen S, Sutherland M, Pipe SW, et al. WFH Guidelines for the Management of Hemophilia, 3rd edition. Haemophilia 2020;26:1-158. https://doi.org/10.1111/hae.14046.

6. Biggs R, Douglas AS, Macfarlane RG, Dacie JV, Pitney WR, Merskey. Christmas disease: a condition previously mistaken for haemophilia. Br Med J 1952;2:1378-1382. https://doi. org/10.1136/bmj.2.4799.1378.

7. Konkle BA, Huston $\mathbf{H}$, Nakaya Fletcher S. Hemophilia A. GeneReviews ${ }^{\circledR}$. Seattle (WA): University of Washington; 2017. Acceso 10 de mayo de 2021. Disponible en https://www. ncbi.nlm.nih.gov/books/NBK1404/.

8. Miller $\mathbf{C H}$, Bean $\mathbf{C J}$. Genetic causes of haemophilia in women and girls. Haemophilia 2021;27:e164-179. https://doi.org/10.1111/ hae. 14186 .

9. Kizilocak $\mathbf{H}$, Young G. Diagnosis and treatment of hemophilia. Clin Adv Hematol Oncol 2019;17:344-351.

10. Cervera-Bravo A. Fisiopatología y trastornos de la coagulación hereditarios más frecuentes. Pediatr Integral 2012;XVI:387-398.

11. Ferreira $\mathbf{C N}$, de Oliveira-Sousa $M$, Sant'AnaDusse LM, Carvalho Md. O novo modelo da cascata de coagulação baseado nas superfícies celulares e suas implicações. Rev Bras Hematol Hemoter 2010;32:416-421.

12. Blanchette VS, Key NS, Ljung LR, MancoJohnson MJ, van den Berg HM, Srivastava A. Definitions in hemophilia: communication from the SSC of the ISTH. J Thromb Haemost 2014;12:1935-1939. https://doi.org/10.1111/ jth.12672.

13. Aghighi $S$, Riddell A, Lee CA, Brown SA, Tuddenham E, Chowdary P. Global coagula- tion assays in hemophilia A: A comparison to conventional assays. Res Pract Thromb Haemost 2020;4:298-308. https://doi.org/10.1002/ rth2.12295

14. van den Berg HM, De Groot PH, Fischer K. Phenotypic heterogeneity in severe hemophilia. J Thromb Haemost 2007;5:S151-156. https:// doi.org/10.1111/j.1538-7836.2007.02503.x.

15. Hoyer LW. Hemophilia A. N Engl J Med 1994;330:38-47. https://doi.org/10.1056/ nejm199401063300108.

16. Acharya SS, Kaplan RN, Macdonald D, Fabiyi OT, DiMichele D, Lyden D. Neoangiogenesis contributes to the development of hemophilic synovitis. Blood 2011;117:2484-2493. https:// doi.org/10.1182/blood-2010-05-284653.

17. Jansen NW, Roosendaal G, Lafeber FP. Understanding haemophilic arthropathy: an exploration of current open issues. $\mathrm{Br} \mathrm{J}$ Haematol 2008;143:632-640. https://doi. org/10.1111/j.1365-2141.2008.07386.x.

18. Witmer C, Presley R, Kulkarni R, Soucie JM, Manno CS, Raffini L. Associations between intracranial haemorrhage and prescribed prophylaxis in a large cohort of haemophilia patients in the United States. Br J Haematol 2011;152:211-216. https://doi.org/10.1111/ j.1365-2141.2010.08469.x.

19. McKusick VA, Hamosh A. Hemophilia A. Baltimore, USA: Online Mendelian Inheritance in Man (OMIM); 2018. Acceso 13 de mayo de 2021. Disponible en https://omim.org/entry/306700.

20. Attard C, van der Straaten $T$, Karlaftis V, Monagle $\mathbf{P}$, Ignjatovic V. Developmental hemostasis: age-specific differences in the levels of hemostatic proteins. J Thromb Haemost 2013;11:18501854. https://doi.org/10.1111/jth.12372.

21. Sato K, Katori N, Suga $Y$, Kiyama S, Uezono S. Coagulation assessment with thromboelastography during abdominal endovascular aneurysm repair in a patient with hemophilia A. JA Clinical Reports 2020;6:7. https://doi.org/10.1186/ s40981-020-0316-0.

22. Rodeghiero F, Tosetto A, Abshire T, Arnold DM, Coller B, James P, et al. ISTH/SSC bleeding assessment tool: a standardized questionnaire and a proposal for a new bleeding score for inherited bleeding disorders. J Thromb Haemost 
2010;8:2063-2065. https://doi.org/10.1111/ j.1538-7836.2010.03975.x.

23. Srivastava A. Diagnosis of haemophilia and other inherited bleeding disorders Is a new paradigm needed? Haemophilia 2021;27:s14-20. https://doi.org/10.1111/ hae.14042.

24. Borhany M, Fatima N, Abid M, Shamsi T, Othman M. Application of the ISTH bleeding score in hemophilia. Transfus Apher Sci 2018;57:556-560. https://doi.org/10.1016/j.transci.2018.06.003.

25. Gouw SC, van den Berg HM, Oldenburg J, Astermark J, de Groot PG, Margaglione M, et al. F8 gene mutation type and inhibitor development in patients with severe hemophilia A: systematic review and meta-analysis. Blood 2012;119:2922-2934. https://doi.org/10.1182/ blood-2011-09-379453.

26. Bagnall RD, Waseem N, Green PM, Giannelli

F. Recurrent inversion breaking intron 1 of the factor VIII gene is a frequent cause of severe hemophilia A. Blood 2002;99:168-174. https:// doi.org/10.1182/blood.v99.1.168.

27. Giancarlo C, Davide M. Hemophilia A and B: molecular and clinical similarities and differences. Haematologica 2019;104:1702-1709. https:// doi.org/10.3324/haematol.2019.221093

28. Plug I, Mauser-Bunschoten EP, BröckerVriends AH, van Amstel HK, van der Bom JG, van Diemen-Homan JE, et al. Bleeding in carriers of hemophilia. Blood 2006;108:52-56. https://doi.org/10.1182/blood-2005-09-3879.

29. Paroskie A, Gailani $D$, DeBaun $M R$, Sidonio RF, Jr. A cross-sectional study of bleeding phenotype in haemophilia A carriers. Br J Haematol 2015;170:223-228. https://doi.org/10.1111/ bjh.13423.

30. Mannucci PM. Back to the future: a recent history of haemophilia treatment. Haemophilia 2008;14:S10-18. https://doi.org/10.1111/ j.1365-2516.2008.01708.x.

31. Franchini M, Mannucci PM. Past, present and future of hemophilia: a narrative review. Orphanet J Rare Dis 2012;7:24. https://doi. org/10.1186/1750-1172-7-24.

32. Mannucci PM, Tuddenham EG. The hemophilias--from royal genes to gene therapy. N Engl J Med 2001;344:1773-1779. https://doi. org/10.1056/nejm200106073442307.
33. Kempton $\mathbf{C L}$, Makris $\mathbf{M}$, Holme PA. Management of comorbidities in haemophilia. Haemophilia 2021;27:s37-45. https://doi.org/10.1111/ hae.14013.

34. Pipe SW, Kaczmarek R, Srivastava A, Pierce GF, Makris M, Hermans C. Management of COVID-19-associated coagulopathy in persons with haemophilia. Haemophilia 2021;27:41-48 https://doi.org/10.1111/hae.14191.

35. Franchini $\mathbf{M}$. The modern treatment of haemophilia: a narrative review. Blood Transfus 2013;11:178182. https://doi.org/10.2450/2012.0166-11.

36. Fuenmayor-Castaño A, Jaramillo-Restrepo M, Salinas-Durán F. Calidad de vida en una población con hemofilia: estudio de corte transversal en un centro de tratamiento de hemofilia. Rev Colomb Reumatol 2017;24:18-24. https:// doi.org/10.1016/j.rcreu.2016.10.006.

37. Shima M, Hanabusa H, Taki M, Matsushita $T$, Sato T, Fukutake $\mathbf{K}$, et al. Factor VIII-mimetic function of humanized bispecific antibody in hemophilia A. N Engl J Med 2016;374:2044-2053. https://doi.org/10.1056/NEJMoa1511769.

38. Zhao Y, Weyand AC, Shavit JA. Novel treatments for hemophilia through rebalancing of the coagulation cascade. Pediatr Blood Cancer 2021;68:e28934. https://doi.org/10.1002/ pbc.28934.

39. Petersen LC. Hemostatic properties of a TFPI antibody. Thromb Res 2012;129:S44-45. https:// doi.org/10.1016/j.thromres.2012.02.030.

40. Shapiro AD, Angchaisuksiri $P$, Astermark J, Benson G, Castaman G, Chowdary P, et al. Subcutaneous concizumab prophylaxis in hemophilia A and hemophilia A/B with inhibitors: phase 2 trial results. Blood 2019;134:1973-1982. https://doi.org/10.1182/ blood.2019001542.

41. Croteau SE, Wang M, Wheeler AP. 2021 clinical trials update: Innovations in hemophilia therapy. Am J Hematol 2021;96:128-144. https:// doi.org/10.1002/ajh.26018.

42. Pierce GF. Uncertainty in an era of transformative therapy for haemophilia: Addressing the unknowns. Haemophilia 2021;27:s103-113. https://doi.org/10.1111/hae.14023.

43. Pipe SW. Gene therapy for hemophilia. Pediatr Blood Cancer 2018;65. https://doi. org/10.1002/pbc.26865. 
44. Peyvandi F, Garagiola I, Young G. The past and future of haemophilia: diagnosis, treatments, and its complications. Lancet 2016;388:187-197. https://doi.org/10.1016/ s0140-6736(15)01123-x.

45. Cannavò A, Valsecchi C, Garagiola I, Palla R, Mannucci PM, Rosendaal FR, et al. Nonneutralizing antibodies against factor VIII and risk of inhibitor development in severe hemophilia A. Blood 2017;129:1245-1250. https://doi. org/10.1182/blood-2016-06-720086.

46. Gringeri A, Mantovani LG, Scalone L, Mannucci PM. Cost of care and quality of life for patients with hemophilia complicated by inhibitors: the COCIS Study Group. Blood 2003;102:2358-2363. https://doi.org/10.1182/ blood-2003-03-0941.

47. Mancuso ME, Mahlangu JN, Pipe SW. The changing treatment landscape in haemophilia: from standard half-life clotting factor concentrates to gene editing. Lancet 2021;397:630-640. https://doi.org/10.1016/ s0140-6736(20)32722-7.

48. Lim MY, Cheng D, Recht M, Kempton CL, Key NS. Management of inhibitors in persons with non-severe hemophilia $A$ in the United States. Am J Hematol 2021;96:e9-11. https://doi. org/10.1002/ajh.26011.

49. Gallo-Durán S, Castillo-Jiménez A, Villalobos-Alvarado G, Caro-Pizarro V, Arroyo-Quirós A. Hemofilia, una revisión de la literatura. Cron Cient 2020;14:6-21.

50. Franchini M, Veneri D, Lippi G. Inherited factor XI deficiency: a concise review. Hematology 2006;11:307-309. https://doi. org/10.1080/10245330600921964.

51. Andreas $T$, Peter $C$, Paul $K$, Jerome $T$, Craig K, Midori S, et al. International recommendations on the diagnosis and treatment of acquired hemophilia A. Haema- tologica 2020;105:1791-1801. https://doi. org/10.3324/haematol.2019.230771.

52. Franchini $\mathbf{M}$, Schiavulli $\mathbf{M}$, Liumbruno GM. Hemostatic therapy as a management strategy for acquired hemophilia: what does the future hold? Expert Rev Hematol 2021;14:263-270. https:// doi.org/10.1080/17474086.2021.1892483.

53. Liga Colombiana de Hemofílicos y Otras Deficiencias Sanguíneas. ColHemofílicos. Bogotá, Colombia: Liga Colombiana de Hemofílicos y Otras Deficiencias Sanguíneas; 2021. Acceso 13 de mayo de 2021. Disponible en http://colhemofilicos.org.co/.

54. Acuña-Merchán $L$, Soler LA, Valderrama F, Daza MT, Fuentes JC. Situación de la hemofilia en Colombia 2017. Cuenta de alto costo, Fondo Colombiano de Enfermedades de Alto Costo. Bogotá, Colombia: Fondo Colombiano de Enfermedades de Alto Costo; 2018. Acceso 12 de mayo de 2021. Disponible en http:// colhemofilicos.org.co/index.php/eventoscolhemofilicos/situacion-de-la-hemofilia-encolombia-2017.

55. Sánchez-Duque JA. Características clínicas y epidemiológicas de pacientes con hemofilia del eje cafetero, Colombia. Rev Cient Cien Med 2017;20:15-20. https://doi.org/10.51581/ rccm.v20i2.117.

56. Mijares ME, Boadas de Sánchez A. Tratamiento profiláctico en la hemofilia en países de la región Latinoamericana. Un reporte del Grupo Latinoamericano para el Impulso del Tratamiento de la Hemofilia (GLAITH). Invest Clin 2015;56:264-275.

57. Martínez-Sánchez LM, Álvarez-Hernández LF, Ruiz-Mejía C, Jaramillo-Jaramillo LI, Builes-Restrepo LN, Villegas-Álzate JD. Hemofilia: abordaje diagnóstico y terapéutico. Revisión bibliográfica. Rev Fac Nac Salud Pública 2018;36:85-93. 